MADPH-99-1123

hep-ph/9906508

\title{
A gauge-mediated supersymmetry breaking model with an extra singlet Higgs field
}

\author{
Tao Han, Danny Marfatia and Ren-Jie Zhang \\ Department of Physics, University of Wisconsin \\ 1150 University Avenue, Madison, WI 53706, USA
}

(August 1999)

\begin{abstract}
We study in some detail the next-to-minimal supersymmetric standard model with gauge mediation of supersymmetry breaking. We find that it is feasible to spontaneously generate values of the Higgs mass parameters $\mu$ and $B_{\mu}$ consistent with radiative electroweak symmetry breaking. The model has a phenomenologically viable particle spectrum. Messenger sneutrinos with mass in the range 6 to $25 \mathrm{TeV}$ can serve as cold dark matter. It is also possible to evade the cosmological domain wall problem in this scenario.
\end{abstract}




\section{INTRODUCTION}

The Standard Model (SM) fulfills electroweak symmetry breaking (EWSB) by introducing a scalar Higgs particle. The mass-squared of this particle suffers from quadratically divergent radiative corrections, and is therefore sensitive to physics effects in the ultraviolet regime, possibly at the grand unification scale $\left(M_{G U T}\right)$ or the Planck scale $\left(M_{P l}\right)$. Stabilizing the electroweak scale against radiative corrections is the primary motivation for exploring theories beyond the SM, among which weak-scale supersymmetry (SUSY) has been the leading candidate [1].

In the minimal supersymmetric extension of the standard model (MSSM), the Higgs scalar potential has the form

$$
V \supset\left(\mu^{2}+m_{H_{u}}^{2}\right)\left|H_{u}\right|^{2}+\left(\mu^{2}+m_{H_{d}}^{2}\right)\left|H_{d}\right|^{2}-\left(B_{\mu} H_{u} H_{d}+\text { h.c. }\right)+\ldots
$$

where $H_{u}$ and $H_{d}$ are two Higgs doublets coupled to the up and down type fermions respectively, $m_{H_{u, d}}$ and $B_{\mu}$ are soft SUSY breaking parameters, and $\mu$ is a mass parameter in the superpotential

$$
W_{\mu} \supset \mu H_{u} H_{d}
$$

To achieve correct electroweak symmetry breaking without drastic fine-tuning, all these mass parameters should have the size of $\mathcal{O}\left(M_{Z}\right)$. The soft parameters can be made to satisfy this requirement. The $\mu$ parameter on the other hand, should naively be of the order of the fundamental scale of the theory like $M_{P l}$ or $M_{G U T}$ since it is a dimensionful parameter of the superpotential. This apparent mismatch is termed as the SUSY $\mu$-problem.

There are many approaches to resolving the $\mu$-problem [2, 3], the simplest one being the introduction of an extra singlet Higgs field $N$. The Higgs superpotential is then written as

$$
W_{N} \supset \lambda_{N} N H_{u} H_{d}-\frac{k}{3} N^{3} .
$$

This is called the next-to-minimal supersymmetric standard model (NMSSM) [2]. The bilinear term $\mu H_{u} H_{d}$ is absent by invoking a discrete $\mathbf{Z}_{\mathbf{3}}$ symmetry under which every chiral superfield $\Phi$ transforms as $\Phi \rightarrow e^{2 \pi i / 3} \Phi$. When $N$ acquires a vacuum expectation value $(\mathrm{VEV})\langle N\rangle \approx \mathcal{O}\left(M_{Z}\right)$, the $\mu$-parameter is effectively generated as

$$
\mu=\lambda_{N}\langle N\rangle \sim \mathcal{O}\left(M_{Z}\right)
$$

The inclusion of the $N^{3}$ term in Eq. (3) is important since without it, the superpotential would have a problematic Peccei-Quinn symmetry. The spontaneous breaking of the $\mathbf{Z}_{\mathbf{3}}$ symmetry by $\langle N\rangle$ leads to the well-known cosmological domain wall problem [⿴囗十 . Also, one should be aware of the potential problem of destabilizing the electroweak scale from tadpoles associated with a gauge singlet [5].

In gauge-mediated supersymmetry breaking models [6] 8], the SUSY breaking in the secluded sector is usually parameterized by a SM singlet chiral superfield $S$ with non-vanishing VEVs for the lowest and highest components $\langle S\rangle+\theta^{2} F_{S}$. If the superpotential contains the term 


$$
W_{S} \supset \lambda_{S} S H_{u} H_{d}
$$

then $\mu=\lambda_{S}\langle S\rangle$ and $B_{\mu}=\lambda_{S} F_{S}$, which implies

$$
B_{\mu} \simeq \mu \Lambda
$$

where $\Lambda=F_{S} /\langle S\rangle \sim \mathcal{O}(100 \mathrm{TeV})$. Therefore $\mu$ and $\sqrt{B_{\mu}}$ cannot both be $\mathcal{O}\left(M_{Z}\right)$. This is known as the $\mu$-problem in GMSB. Some solutions have been proposed in [9, 10. In particular, Ref. 10 studied the GMSB paradigm with the NMSSM, but the conclusion is negative if one does not introduce extra vector-like quarks in the theory.

In this paper we perform a detailed analysis of a gauge-mediated model with an extra singlet Higgs field in a most general superpotential including all couplings between the singlets, messengers and Higgs fields, respecting the $\mathbf{Z}_{\mathbf{3}}$ symmetry. Without constructing a SUSY breaking model explicitly, we consider it as a phenomenological model and study its consistency with theoretical and experimental constraints. In Section II we describe our general framework for the gauge-mediated SUSY breaking model with an extra singlet. In Section III we present a numerical study of this model, concentrating on the $\mu$-problem and phenomenologically viable mass spectrum of SUSY particles (sparticles) with emphasis on the next-to-the-lightest supersymmetric particle (NLSP) and Higgs bosons. In contrast to the conclusion of Ref. [10], we find that suitable $\mu$ and $B_{\mu}$ parameters can be generated in this minimal scenario with all phenomenological constraints satisfied. In Section IV we show that messenger sneutrinos can serve as a cold dark matter candidate and that they are sufficiently massive to evade the current experimental bound from direct searches. In Section $\mathrm{V}$ we demonstrate that the domain wall problem may find a solution due to fast decay of the walls induced by higher dimensional operators, yet consistent with EWSB. We conclude in Section VI. Some technical details of the model-the renormalization group equations (RGEs) and Higgs boson mass matrices - are provided in two appendices.

\section{THE MINIMAL GMSB WITH AN EXTRA SINGLET}

In our phenomenological approach to the GMSB, we parameterize SUSY breaking in the secluded sector by a SM gauge singlet spurion $S=\langle S\rangle+\theta^{2} F_{S}$. The SUSY breaking is then transmitted to the observable sector via vector-like messengers $\Phi$ and $\bar{\Phi}$ which couple to $S$ according to $S \bar{\Phi} \Phi$. In the simplest case, $\Phi$ and $\bar{\Phi}$ transform as a single flavor of $\mathbf{5}+\overline{\mathbf{5}}$ of $\mathrm{SU}(5)$.

In gauge-mediated models not involving any direct messenger-matter interactions, it is not possible to generate $\mu$ and $B_{\mu}$ consistent with EWSB. In fact, even if the spurion $S$ couples directly to the Higgs superfields as in Eq. (5), one can easily show that $\mu=\lambda_{S}\langle S\rangle$ and $B_{\mu}=\lambda_{S} F_{S}$, which leads to the troublesome relation in Eq. (6). This is a general result irrespective of whether $\mu$ is generated spontaneously or radiatively. One cannot get both $\mu$ and $\sqrt{B_{\mu}}$ of the order of $M_{Z}$ and EWSB fails because the large value of $B_{\mu}$ destabilizes the scalar potential.

Motivated by the NMSSM model, we consider a minimal gauge-mediated model with an extra singlet Higgs field. The most general superpotential respecting a $\mathbf{Z}_{\mathbf{3}}$ symmetry is

$$
W=\xi_{S} S \bar{\Phi} \Phi+\xi_{N} N \bar{\Phi} \Phi-\frac{\eta_{S}}{2} S^{2} N-\frac{\eta_{N}}{2} N^{2} S+\lambda_{S} S H_{u} H_{d}+\lambda_{N} N H_{u} H_{d}-\frac{k}{3} N^{3} .
$$


The gauginos and scalars acquire mass radiatively at the 1-loop and 2-loop level respectively, and have the following form

$$
\begin{aligned}
& M_{i} \simeq k_{i} \frac{\alpha_{i}}{4 \pi} \Lambda, \\
& m_{\tilde{f}}^{2} \simeq 2 \sum_{i=1}^{3} C_{i}^{\tilde{f}} k_{i}\left(\frac{\alpha_{i}}{4 \pi} \Lambda\right)^{2},
\end{aligned}
$$

where $k_{1}=5 / 3, k_{2}=k_{3}=1$, and $C_{1}^{\tilde{f}}=Y^{2}$ for $\mathrm{U}(1), C_{2}^{\tilde{f}}=3 / 4$ for weak $\mathrm{SU}(2)$ doublets and $C_{3}^{\tilde{f}}=4 / 3$ for color triplets. $\Lambda=F_{S} / S$ is the effective SUSY-breaking scale. In models in which messengers and matter do not interact directly, the trilinear soft $A$-terms and $m_{N}^{2}$ arise only at 2-loop and 3-loop respectively, and can be neglected. The superpotential Eq. (17) contains messenger-matter couplings, thus inducing $A_{\lambda, k}$ and $m_{N}^{2}$ at 1-loop and 2-loop respectively [8],

$$
\begin{aligned}
\frac{A_{k}}{3} & =A_{\lambda_{N}} \simeq-5 \frac{\alpha_{\xi_{N}}}{4 \pi} \Lambda \\
m_{N}^{2} & \simeq 10 \alpha_{\xi_{N}}\left(\frac{7}{2} \alpha_{\xi_{N}}-\frac{8}{5} \alpha_{3}-\frac{3}{5} \alpha_{2}-\frac{13}{3} \alpha_{1}\right)\left(\frac{\Lambda}{4 \pi}\right)^{2},
\end{aligned}
$$

where $\alpha_{\xi_{N}}=\xi_{N}^{2} / 4 \pi$. Note that the contributions from the gauge couplings can make $m_{N}^{2}$ negative for small values of $\xi_{N}$. Among the couplings in Eq. (7), we may anticipate that $\xi_{S}, \xi_{N}, \lambda_{N}, k \sim \mathcal{O}(1)$ and that $\eta_{N}, \eta_{S}$ and $\lambda_{S}$ will be small due to the large VEV from $S$. We will determine the pattern for phenomenologically viable solutions. The only result contrary to these expectations is that we find $\eta_{N} \sim \mathcal{O}(1)$. We will not explore the possible symmetries in terms of model-building that naturally lead to this pattern [6,7,10].

The relevant part of the scalar potential is

$$
V_{\text {Higgs }}=V_{F}+V_{D}+V_{\text {tadpole }}+V_{\text {soft }}+\Delta V
$$

where

$$
\begin{aligned}
& V_{F}=\left|\lambda_{S} S+\lambda_{N} N\right|^{2}\left(\left|H_{u}\right|^{2}+\left|H_{d}\right|^{2}\right)+\left|\lambda_{N} H_{u} H_{d}-k N^{2}-\frac{\eta_{S}}{2} S^{2}-\eta_{N} N S\right|^{2} \\
& \quad+\left|\lambda_{S} H_{u} H_{d}+F_{S}-\frac{\eta_{N}}{2} N^{2}-\eta_{S} N S\right|^{2}, \\
& V_{D}=\frac{g_{2}^{2}}{8}\left(H_{u}^{\dagger} \vec{\sigma} H_{u}+H_{d}^{\dagger} \vec{\sigma} H_{d}\right)^{2}+\frac{g^{\prime 2}}{8}\left(\left|H_{u}\right|^{2}-\left|H_{d}\right|^{2}\right)^{2}, \\
& V_{\text {tadpole }}=\frac{\xi_{S} \xi_{N}}{8 \pi^{2}} \frac{N F_{S}^{2}}{S}, \\
& V_{\text {soft }}=m_{H_{u}}^{2}\left|H_{u}\right|^{2}+m_{H_{d}}^{2}\left|H_{d}\right|^{2}+m_{N}^{2}|N|^{2}-\left(\lambda_{N} A_{\lambda_{N}} N H_{u} H_{d}+\frac{k A_{k}}{3} N^{3}+h . c .\right), \\
& \Delta V=\frac{3}{32 \pi^{2}}\left[m_{\tilde{t}_{1}}^{4}\left(\ln \frac{m_{\tilde{t}_{1}}^{2}}{Q^{2}}-\frac{3}{2}\right)+m_{\tilde{t}_{2}}^{4}\left(\ln \frac{m_{\tilde{t}_{2}}^{2}}{Q^{2}}-\frac{3}{2}\right)-2 m_{t}^{4}\left(\ln \frac{m_{t}^{2}}{Q^{2}}-\frac{3}{2}\right)\right],
\end{aligned}
$$

where $V_{\text {tadpole }}$ arises from the $N \bar{\Phi} \Phi$ coupling via one-loop tadpole diagrams involving the messenger fields [10]. For the one-loop potential term $\Delta V$, we have only included the dominant contributions from the top-quark and top-squark diagrams. $Q$ is the renormalization scale in the $\overline{D R}$ scheme. 


\section{ANALYSIS OF THE MODEL}

\section{A. Numerical Procedure}

To study physics at the electroweak scale, we perform a one-loop renormalization group (RG) evolution analysis. The relevant RGEs can be derived from [11] and are listed in Appendix A.

We fix the boundary conditions for the RG evolution as follows: At the messenger scale, we impose the conditions in Eqs.(8)-(11). The coupling constant $\xi_{S}$ is fixed at the messenger scale to be unity. $\xi_{N}, \eta_{S}$ and $\eta_{N}$ are solved at the electroweak scale by minimizing the potential as described below. Values of the other three couplings, $\lambda_{S}, \lambda_{N}$ and $k$ are chosen randomly at the electroweak scale. We require the couplings to be in the perturbative ranges

$$
-1 \leq \xi_{N}(S) \leq 1, \quad 0 \leq \lambda_{S}, \lambda_{N}, \eta_{N} \leq 1, \quad 0 \leq k \leq 0.65,
$$

where the upper bound on $k$ comes from the demand that it not hit the Landau pole below the GUT scale. If we require that $k$ be perturbative only below the messenger scale $\Lambda$ where unspecified new physics sets in, we find $k \leq 1.36$. It is found to be unnecessary to place bounds on the value of $\eta_{S}$. In the numerical analysis, we will neglect Yukawa coupling terms in the RGEs, except for those involving the top quark $\left(h_{t}\right)$. The $A$-terms run slowly and so can be approximated by their boundary values. All RGEs are run down to $Q=\sqrt{m_{\tilde{t}_{1}} m_{\tilde{t}_{2}}}$.

In the RGE running, $m_{H_{u}}^{2}$ receives large correction from the heavy stop loop through the Yukawa coupling $h_{t}$,

$$
m_{H_{u}}^{2}(Q) \simeq m_{H_{u}}^{2}(S)-\frac{3}{8 \pi^{2}} h_{t}^{2}\left(m_{\tilde{t}_{L}}^{2}(S)+m_{\tilde{t}_{R}}^{2}(S)+m_{H_{u}}^{2}(S)\right) \ln \frac{S}{Q},
$$

and becomes negative at the electroweak scale, triggering EWSB. A satisfactory estimate for $m_{N}^{2}$ at the electroweak scale can be found by approximating the RGE for $m_{N}^{2}$ :

$$
\begin{aligned}
m_{N}^{2}(Q) \simeq m_{N}^{2}(S) & -\frac{\lambda_{N}^{2}}{4 \pi^{2}}\left(2 m_{H_{d}}^{2}(S)-\frac{3}{4 \pi^{2}} h_{t}^{2} m_{\tilde{t}}^{2}(S) \ln \frac{S}{Q}+m_{N}^{2}(S)+A_{\lambda_{N}}^{2}\right) \ln \frac{S}{Q} \\
& -\frac{k^{2}}{4 \pi^{2}}\left(3 m_{N}^{2}(S)+A_{k}^{2}\right) \ln \frac{S}{Q} .
\end{aligned}
$$

which can be either positive or negative depending on the relative values of the couplings.

The physical mass spectrum at the electroweak scale is determined by the five VEVs

$$
\left\langle H_{u}\right\rangle=v_{u}, \quad\left\langle H_{d}\right\rangle=v_{d}, \quad\langle N\rangle=x, \quad\langle S\rangle=y \quad \text { and } \quad F_{S} .
$$

The values of $M_{Z}$ and top-quark mass fix the scale

$$
v \equiv \sqrt{v_{u}^{2}+v_{d}^{2}}=174 \mathrm{GeV}, \quad m_{t} \equiv h_{t} v_{u}=165 \pm 5 \mathrm{GeV}
$$

where we have used the $\overline{D R}$ value of the top-quark running mass. We also require that $\tan \beta=v_{u} / v_{d}$ satisfy

$$
1.5 \leq \tan \beta \leq 50,
$$


which leads to $0.92 \leq h_{t} \leq 1.2$. We impose the following constraints on the other VEVs

$$
100 \leq x \leq 1000 \mathrm{GeV}, \quad 3.8 \times 10^{4} \leq \Lambda, y \leq 10^{5} \mathrm{GeV}, \quad \Lambda \leq 0.9 y,
$$

where $\Lambda=F_{S} / y$. The lower bound on $\Lambda$ arises from the right-handed slepton mass constraint (see below), and the last condition in Eq. (24) is adopted to avoid possible large corrections from $\Lambda \approx y$. The calculated mass spectrum for the sparticles must satisfy the current lower limits from direct experimental searches 12,13

$$
\begin{aligned}
& m_{\tilde{g}}>190 \mathrm{GeV}, \quad m_{\tilde{e}_{R}}>80 \mathrm{GeV}, \\
& m_{h} \equiv m_{S_{1}}>95.5 \mathrm{GeV}, \quad m_{A} \equiv m_{P_{1}}>84.5 \mathrm{GeV}, \quad m_{H^{ \pm}}>77.3 \mathrm{GeV}, \\
& m_{\tilde{\chi}_{1}^{ \pm}}>95 \mathrm{GeV}, \quad m_{\tilde{b}_{1}}>75 \mathrm{GeV}, \\
& \text { if } \frac{m_{\tilde{\chi}_{1}^{0}}}{m_{\tilde{\tau}_{1}}}<1, m_{\tilde{\chi}_{1}^{0}}>55 \mathrm{GeV} ; \quad \text { if } \frac{m_{\tilde{\chi}_{1}^{0}}}{m_{\tilde{\tau}_{1}}}>1, m_{\tilde{\tau}_{1}}>68 \mathrm{GeV} ; \\
& \text { if } \frac{m_{\tilde{\chi}_{1}^{0}}}{m_{\tilde{\tau}_{1}}}>1 \text { and } m_{\tilde{\chi}_{1}^{0}}<87 \mathrm{GeV} \text { then } m_{\tilde{\tau}_{1}}>84 \mathrm{GeV} .
\end{aligned}
$$

The minimization conditions to be imposed at $\mathcal{O}\left(M_{Z}\right)$ can be derived by differentiating Eq. (12) with respect to $v_{d}, v_{u}$ and $x$ :

$$
\begin{aligned}
& 2 m_{H_{d}}^{2} v_{d}+\frac{\bar{g}^{2} v_{d}\left(v_{d}^{2}-v_{u}^{2}\right)}{2}+v_{u} \lambda_{N}\left(-2 k x^{2}-2 x y \eta_{N}-y^{2} \eta_{S}+2 v_{d} v_{u} \lambda_{N}\right)+2 v_{d}\left(x \lambda_{N}+y \lambda_{S}\right)^{2} \\
& \quad+v_{u} \lambda_{S}\left(2 F_{S}-x^{2} \eta_{N}-2 x y \eta_{S}+2 v_{d} v_{u} \lambda_{S}\right)+\partial_{v_{d}} \Delta V=0, \\
& 2 m_{H_{u}}^{2} v_{u}-\frac{\bar{g}^{2} v_{u}\left(v_{d}^{2}-v_{u}^{2}\right)}{2}+v_{d} \lambda_{N}\left(-2 k x^{2}-2 x y \eta_{N}-y^{2} \eta_{S}+2 v_{d} v_{u} \lambda_{N}\right)+2 v_{u}\left(x \lambda_{N}+y \lambda_{S}\right)^{2} \\
& \quad+v_{d} \lambda_{S}\left(2 F_{S}-x^{2} \eta_{N}-2 x y \eta_{S}+2 v_{d} v_{u} \lambda_{S}\right)+\partial_{v_{u}} \Delta V=0, \\
& 2 m_{N}^{2} x+\frac{F_{S}^{2} \xi_{N} \xi_{S}}{8 \pi^{2} y}+\left(2 k x+y \eta_{N}\right)\left(2 k x^{2}+2 x y \eta_{N}+y^{2} \eta_{S}-2 v_{d} v_{u} \lambda_{N}\right)+2 v^{2} \lambda_{N}\left(x \lambda_{N}+y \lambda_{S}\right) \\
& \quad+\left(x \eta_{N}+y \eta_{S}\right)\left(-2 F_{S}+x^{2} \eta_{N}+2 x y \eta_{S}-2 v_{d} v_{u} \lambda_{S}\right)+\partial_{x} \Delta V=0,
\end{aligned}
$$

where $\bar{g}^{2}=g^{\prime 2}+g_{2}^{2}$. Running $\xi_{S}$ down to the electroweak scale, we get $\xi_{S}(Q) \simeq 0.94$. $\eta_{S}, \eta_{N}$ and $\xi_{N}$ are determined from the minimization conditions Eqs. (26)-(28). These equations also lead to the relations

$$
\begin{aligned}
\mu^{2} & =-\frac{M_{Z}^{2}}{2}+\frac{\left[m_{H_{d}}^{2}+\left(\partial_{v_{d}} \Delta V\right) /\left(2 v_{d}\right)\right]-\left[m_{H_{u}}^{2}+\left(\partial_{v_{u}} \Delta V\right) /\left(2 v_{u}\right)\right] \tan ^{2} \beta}{\tan ^{2} \beta-1}, \\
\sin 2 \beta & =\frac{2 B_{\mu}}{\left[m_{H_{d}}^{2}+\left(\partial_{v_{d}} \Delta V\right) /\left(2 v_{d}\right)\right]+\left[m_{H_{u}}^{2}+\left(\partial_{v_{u}} \Delta V\right) /\left(2 v_{u}\right)\right]+2 \mu^{2}},
\end{aligned}
$$

where

$$
\begin{aligned}
\mu & \equiv \lambda_{N} x+\lambda_{S} y \\
B_{\mu} & \equiv \lambda_{N}\left(A_{\lambda_{N}} x+k x^{2}+\eta_{N} x y+\frac{\eta_{S} y^{2}}{2}-\lambda_{N} v_{u} v_{d}\right)+\lambda_{S}\left(-F_{S}+\eta_{S} x y+\frac{\eta_{N} x^{2}}{2}-\lambda_{S} v_{u} v_{d}\right)
\end{aligned}
$$

The parameters $\mu$ and $B_{\mu}$ can be evaluated by either set of equations and they must be consistent with each other since all the parameters have been determined by conditions on EWSB. 
B. Viable solutions, $\mu$ and $B_{\mu}$ parameters
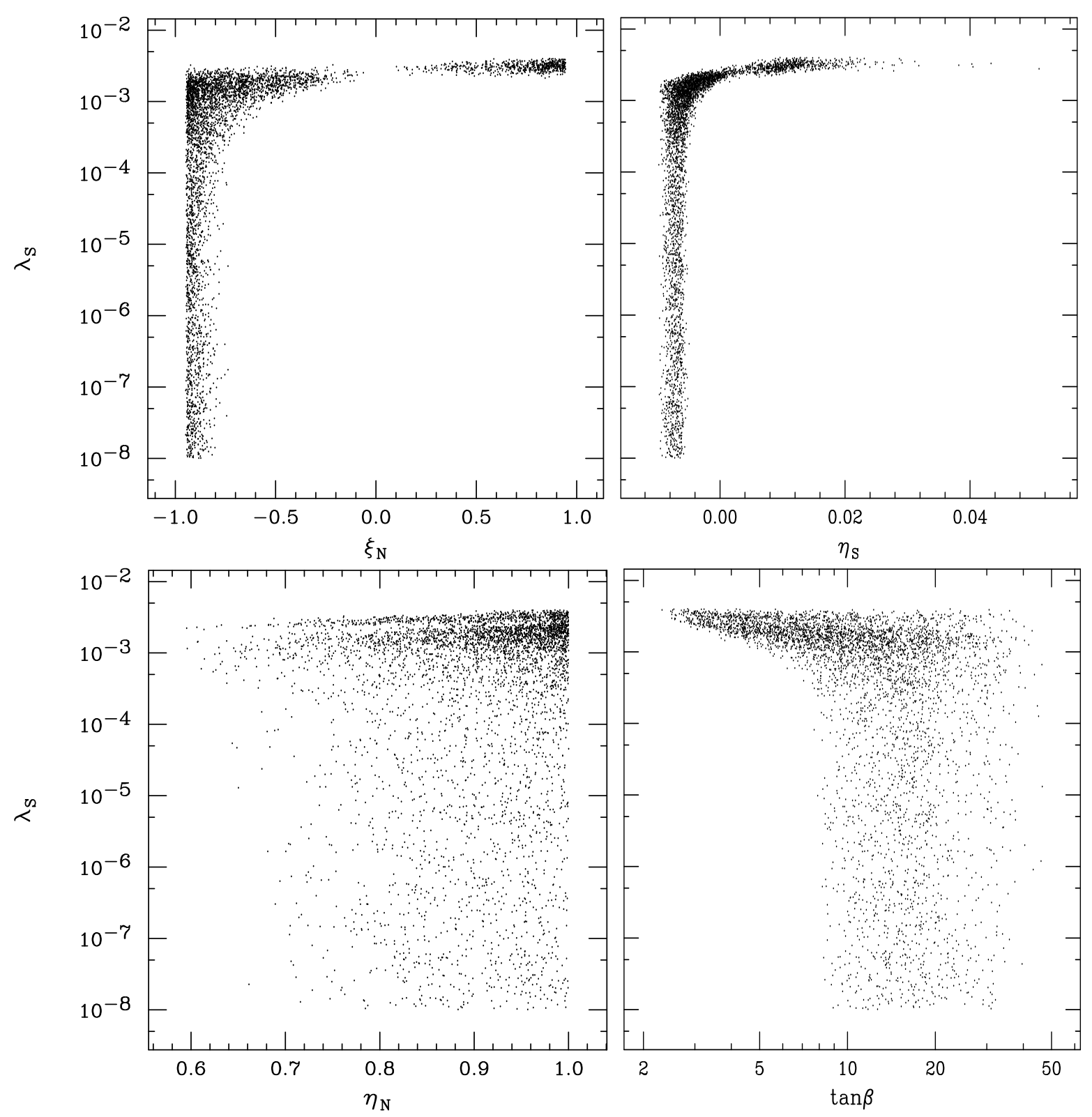

FIG. 1. Phenomenologically viable solutions in the $\lambda_{S^{-}} \xi_{N}, \lambda_{S}-\eta_{S}, \lambda_{S}-\eta_{N}$ and $\lambda_{S}-\tan \beta$ planes.

On inspection of the parameter space of the variables chosen randomly in the allowed ranges in Eq. (18), it is found that there are solutions satisfying the theoretical and experimental constraints of Eqs. (24)-(25). We first note that $\eta_{N}$ and the "NMSSM couplings" $\lambda_{N}$ and $k$ are essentially independent of other parameters and mostly of $\mathcal{O}(1)$,

$$
0.6 \lesssim \eta_{N} \lesssim 1.0, \quad 0.05 \lesssim \lambda_{N} \lesssim 1.0, \quad 0.001 \lesssim k \lesssim 0.65
$$

$\xi_{N}$ and $\eta_{S}$ depend quite crucially on the values of $\lambda_{S}$ and take on values of $\mathcal{O}(1)$ and $\mathcal{O}\left(10^{-3}-10^{-2}\right)$ respectively, 


$$
\begin{aligned}
& -0.95 \lesssim \xi_{N} \lesssim 0.95 \text { and }\left|\xi_{N}\right| \gtrsim 0.1 \\
& -0.01 \lesssim \eta_{S} \lesssim 0.05 \text { and }\left|\eta_{S}\right| \gtrsim 10^{-4}
\end{aligned}
$$

corresponding to

$$
0 \lesssim \lambda_{S} \lesssim 4 \times 10^{-3}
$$

The correlations for those parameters are shown in Fig. 囵. There are two rather distinctive regions in $\lambda_{S}$.

1. Vanishing $\lambda_{S}$ : There are viable solutions for vanishing $\lambda_{S}$. In this case, typically $\lambda_{S} \lesssim 10^{-3}$, leading to tightly constrained solutions for the allowed values of $\xi_{N}$ and $\eta_{S}$.

$$
-0.95 \lesssim \xi_{N} \lesssim-0.3, \quad-0.01 \lesssim \eta_{S} \lesssim-4 \times 10^{-3} .
$$

It is interesting to note that $\eta_{S}$ and $\xi_{N}$ are always negative, thus picking out a particular choice of phases in the superpotential

$$
W \supset \xi_{S} S \Phi \bar{\Phi}-\left|\xi_{N}\right| N \Phi \bar{\Phi}+\frac{\left|\eta_{S}\right|}{2} S^{2} N+\ldots
$$

Also notice that small $\tan \beta$ is excluded.

2. Finite $\lambda_{S}$ : For $\lambda_{S} \gtrsim 10^{-3}$, the couplings $\xi_{N}$ and $\eta_{S}$ can take on the whole range of permissible values. Moreover, small $\tan \beta$ solutions are allowed.

Since we wish to keep all terms in the superpotential that respect the $\mathbf{Z}_{\mathbf{3}}$ symmetry, we avoid a vanishing $\lambda_{S}$ and restrict our attention to the case $\lambda_{S} \geq 10^{-3}$ in the rest of the paper. This choice gives us greater freedom in parameter space.

The parameters $\mu$ and $B_{\mu}$ are spontaneously generated and are of $\mathcal{O}\left(M_{Z}\right)$ and $\mathcal{O}\left(M_{Z}^{2}\right)$, respectively. As shown in Fig. 2(a), the values lie in the ranges

$$
275 \lesssim \mu \lesssim 550 \mathrm{GeV}, \quad 5 \times 10^{3} \lesssim B_{\mu} \lesssim 2 \times 10^{5}(\mathrm{GeV})^{2} .
$$

Moreover the " $B$-parameter" $\left(B_{\mu} / \mu\right)$ familiar from supergravity theories is also $\mathcal{O}\left(M_{Z}\right)$ for most of the parameter space

$$
10 \lesssim \frac{B_{\mu}}{\mu} \lesssim 300 \mathrm{GeV},
$$

as shown in Fig. 2(b). For $\tan \beta \gtrsim 2, M_{Z}^{2} / 2 \simeq-\mu^{2}-m_{H_{u}}^{2}$. A good measure of fine-tuning of the Higgs potential parameters is $M_{Z}^{2} / 2 \mu^{2}$ [14]. The fine-tune is at the $1-6 \%$ level as seen in Fig. 包, where the fine-tune parameter is shown versus $\tan \beta$ and $\Lambda$. This level of fine-tune could be significantly improved if one introduces extra vector-like triplets that couple only to the singlet $N$ [15]. Recall that in the MSSM, $\mu$ of electroweak scale is put in by hand. Even if the very lowest value of $\mu$ (consistent with $m_{\tilde{e}_{R}} \gtrsim 80 \mathrm{GeV}$ ) is used, the best possible fine-tune is $7 \%$. 

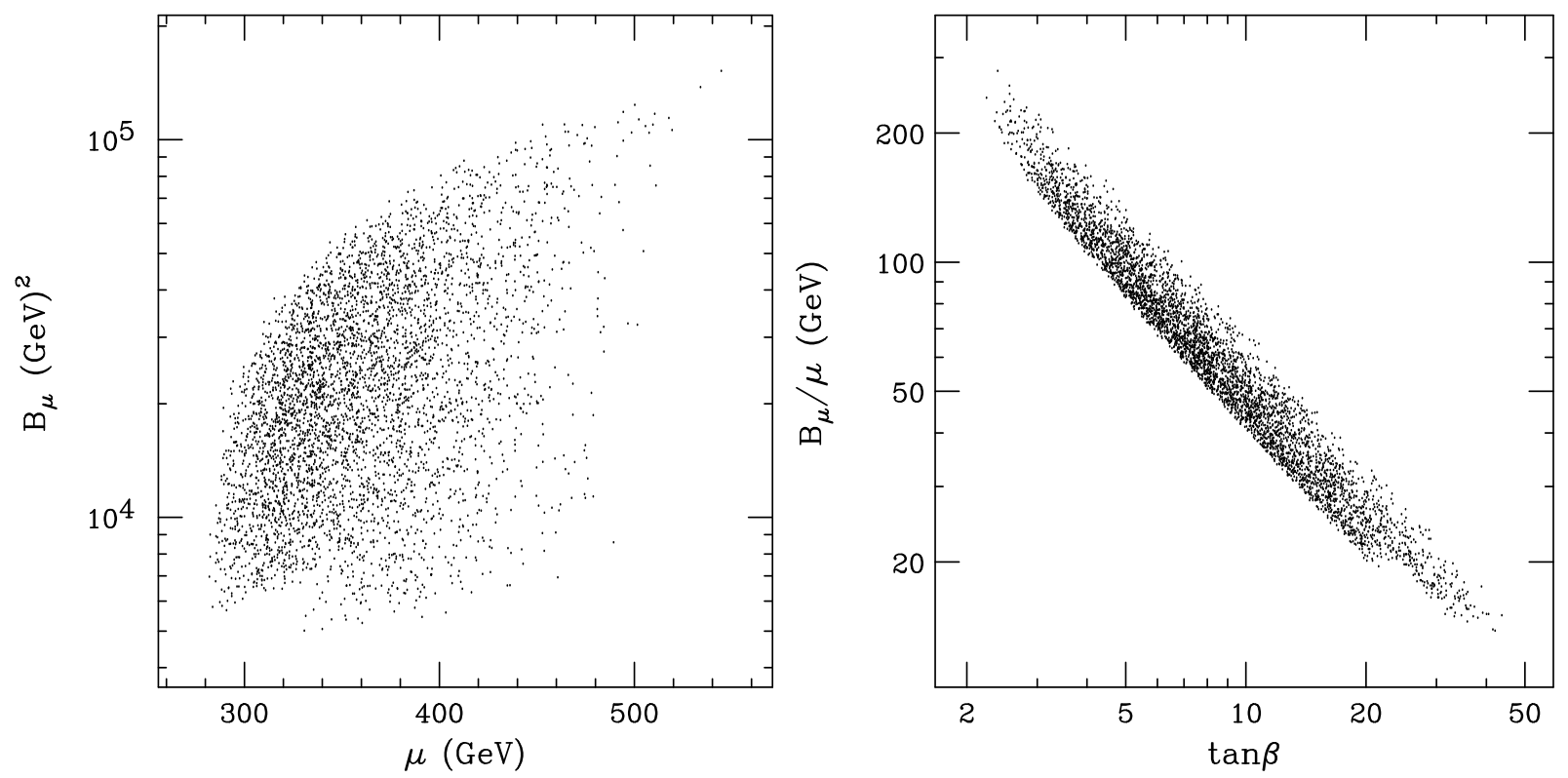

FIG. 2. The allowed region in the (a) $B_{\mu}-\mu$ plane and (b) $\left(B_{\mu} / \mu\right)-\tan \beta$ plane.

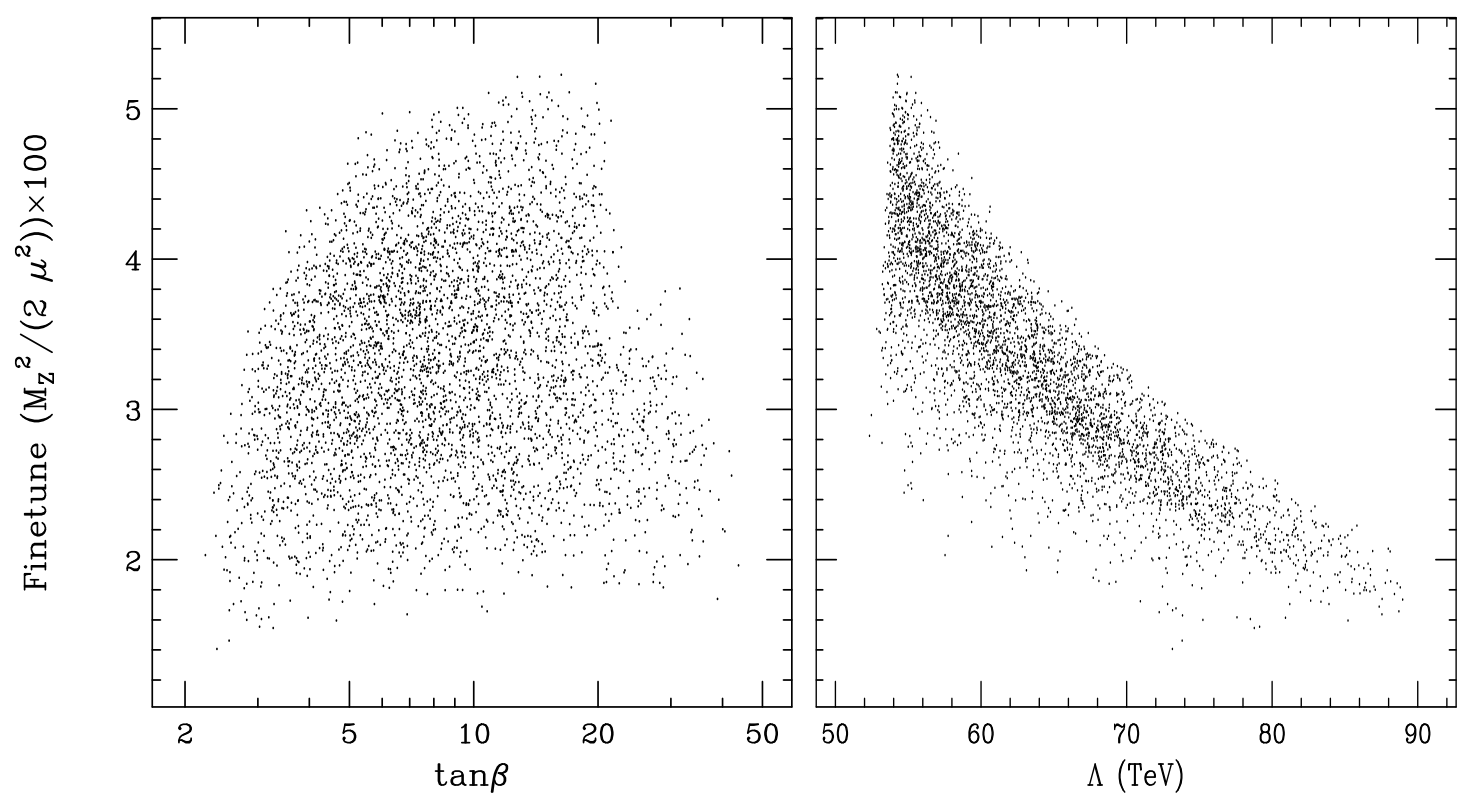

FIG. 3. The percentage fine-tune versus $\tan \beta$ and $\Lambda$. 


\section{Mass Spectrum}

The details of sparticle spectroscopy of models with gauge mediation have been discussed in the literature over quite large ranges of both $\tan \beta$ and $\Lambda$ [16]. Many of those results carryover unchanged to the case at hand. As one would expect, the main differences arise from the specific choice of the Higgs sector superpotential. The mass-squared matrices for the CP-even and CP-odd Higgs bosons and their eigenvalues are presented in the Appendix B.

We find the range for the CP-even Higgs boson masses

$$
95.5 \lesssim m_{S_{1}} \lesssim 130 \mathrm{GeV}, \quad 200 \lesssim m_{S_{2}} \lesssim 650 \mathrm{GeV}, \quad m_{S_{3}} \gtrsim 5 \mathrm{TeV}
$$

We see that $m_{S_{1}}$ has the usual upper bound of $130 \mathrm{GeV}$. If the perturbativity for $k$ is relaxed to the messenger scale, $m_{S_{1}}$ can be as heavy as about $170 \mathrm{GeV}$. In the NMSSM, there are two CP-odd Higgs boson mass eigenstates $\left(P_{1}, P_{2}\right)$. The mass of the lighter CP-odd state lies in the range $340 \lesssim m_{P_{1}} \lesssim 650 \mathrm{GeV}$ indicating that we are well into the decoupling regime [17]. $P_{1}$ is primarily a mixture of $\operatorname{Im} H_{d}$ and $\operatorname{Im} H_{u}$ since the mixing angle $\gamma$ between $A^{0}$ and $\operatorname{Im} N$ is $\mathcal{O}\left(10^{-4}\right)$ (see Appendix B). We also find that the charged Higgs bosons $H^{ \pm}$ are almost degenerate with $P_{1}$.

Since $\mu$ is large compared to $M_{Z}$, the higgsinos and gauginos decouple from each other and the lightest neutralino is mostly bino. It is the $\tilde{\chi}_{1}^{0}$-NLSP for a large region of parameter space

$$
\tan \beta \lesssim 22 \text { or } \Lambda \lesssim 64 \mathrm{TeV}
$$

Due to the mixing in the $\tau$-slepton (stau) mass matrix, $m_{\tilde{\tau}_{1}}$ can be much smaller than $m_{\tilde{e}_{R}, \tilde{\mu}_{R}}$ and becomes the NLSP for large $\tan \beta$ and high values of $\Lambda$. These features are shown in Fig. 4 . We find the mass ranges for the lighter sparticles are

$$
65 \lesssim m_{\tilde{\chi}_{1}^{0}} \lesssim 125 \mathrm{GeV}, \quad 120 \lesssim m_{\tilde{\chi}_{1}^{+}} \lesssim 230 \mathrm{GeV}, \quad 68 \lesssim m_{\tilde{\tau}_{1}} \lesssim 150 \mathrm{GeV}
$$

Gluinos and squarks are much heavier than the electroweak states because they acquire mass mainly from strong interactions. We find the ranges the of stop masses as follows:

$$
650 \lesssim m_{\tilde{t}_{1}} \lesssim 1080 \mathrm{GeV}, \quad 700 \lesssim m_{\tilde{t}_{2}} \lesssim 1140 \mathrm{GeV}
$$

Table gives a representative set of parameters and the corresponding mass spectrum.

\begin{tabular}{c|c|c|c|c|c|c|c|c|c|c|}
\hline \hline $\tan \beta$ & $\xi_{S}$ & $\xi_{N}$ & $\eta_{S}$ & $\eta_{N}$ & $\lambda_{S}$ & $\lambda_{N}$ & $k$ & $\langle S\rangle$ & $\Lambda$ & $\langle N\rangle$ \\
\hline 24.3 & 0.94 & 0.71 & $8.3 \times 10^{-3}$ & 0.87 & $3.0 \times 10^{-3}$ & 0.31 & 0.40 & $97 \mathrm{TeV}$ & $77 \mathrm{TeV}$ & 413 \\
\hline \hline
\end{tabular}

\begin{tabular}{|c|c|c|c|c|c|c|c|c|c|c|c|c|c|}
\hline \hline$\mu$ & $M_{1}$ & $M_{2}$ & $M_{3}$ & $m_{\tilde{\chi}_{1}^{0}}$ & $m_{\tilde{\chi}_{1}^{+}}$ & $m_{S_{1}}$ & $m_{P_{1}}$ & $m_{H^{ \pm}}$ & $m_{\tilde{\tau}_{1}}$ & $m_{\tilde{\tau}_{2}}$ & $m_{\tilde{t}_{1}}$ & $m_{\tilde{t}_{2}}$ & $m_{\phi^{0}}$ \\
\hline \hline 421 & 107 & 208 & 649 & 106 & 196 & 122 & 500 & 505 & 109 & 288 & 949 & 982 & $10.7 \mathrm{TeV}$ \\
\hline \hline
\end{tabular}

TABLE I. A representative set of parameters and the mass spectrum. All masses are in $\mathrm{GeV}$ unless specified. 

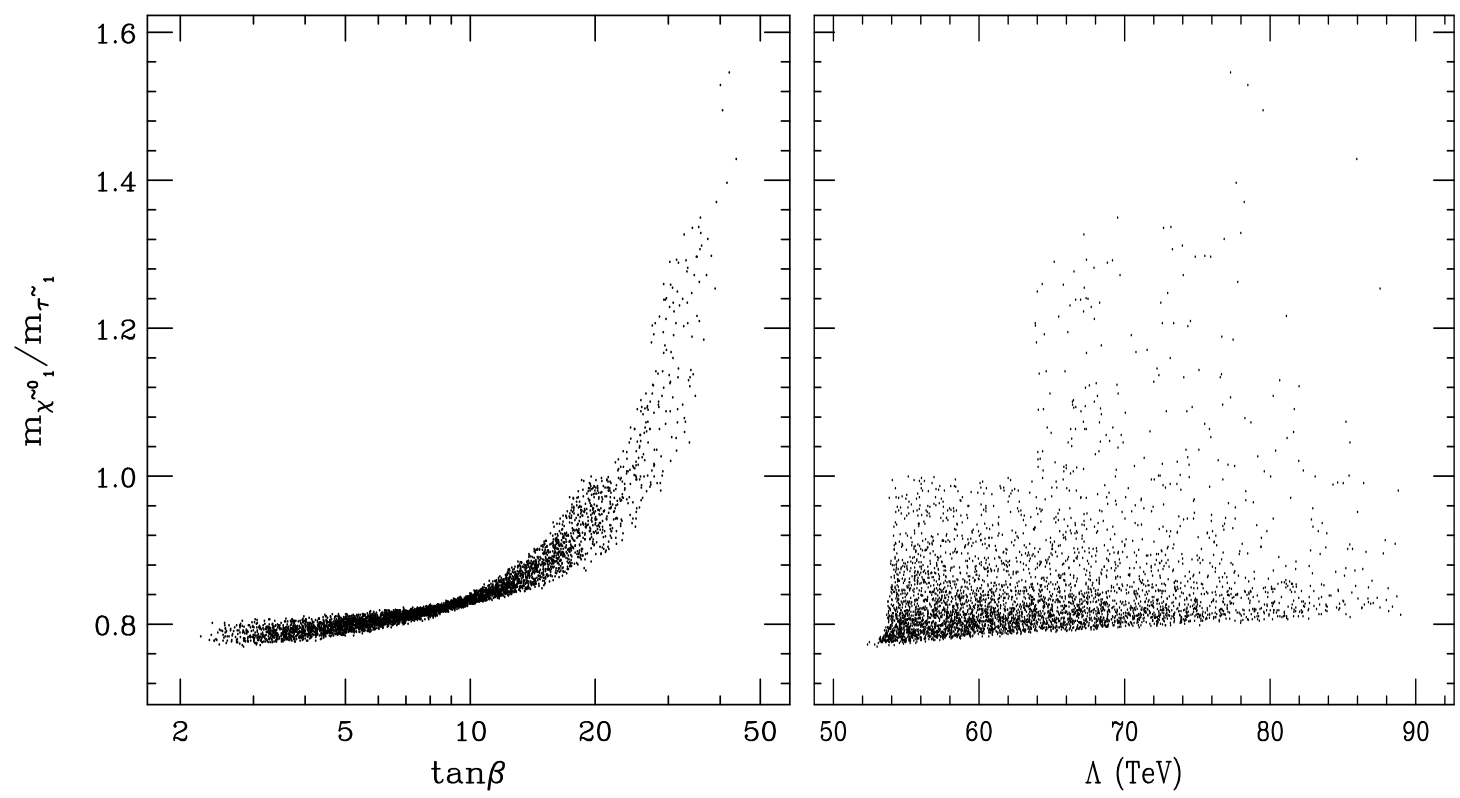

FIG. 4. Regions of $\tilde{\chi}_{1}^{0}$-NLSP and $\tilde{\tau}_{1}$-NLSP in the $\left(m_{\tilde{\chi}_{1}^{0}} / m_{\tilde{\tau}_{1}}\right)-\tan \beta$ and $\left(m_{\tilde{\chi}_{1}^{0}} / m_{\tilde{\tau}_{1}}\right)-\Lambda$ planes.

\section{MESSENGER SNEUTRINOS AS COLD DARK MATTER}

The possibility of messenger sneutrinos $\left(\phi^{0}\right)$ as a cold dark matter (CDM) candidate was investigated in the context of the minimal GMSB model in Refs. [18,19. Requiring that the Universe not be over-closed by the messenger sneutrinos $\left(\Omega_{\phi^{0}} h^{2}<1\right)$ puts an upper bound on their masses

$$
m_{\phi^{0}}<3 \mathrm{TeV} \text {. }
$$

It certainly requires some degree of fine-tuning to obtain such a light messenger particle as the messenger scale is about $50-100 \mathrm{TeV}$. More severely, a scalar DM particle with mass lighter than $3 \mathrm{TeV}$ and with SM interactions has already been experimentally ruled out [20]. In the GMSB model with an extra singlet Higgs field, additional contributions from the singlet $N$ can sufficiently increase the annihilation cross-section of $\phi^{0}$, and therefore loosen the above bound.

We perform a relic density calculation in our model. We first note that the freezeout temperature $T_{f} \simeq m_{\phi^{0}} / 20 \gg m_{\phi^{-}}-m_{\phi^{0}}$, which implies that both charged and neutral components are present in the thermal bath at $T_{f}$ [19. We calculate the relic density in the limit of symmetric phase for the four components $\left(\phi^{Q}, \phi^{Q \dagger}\right.$ with $\left.Q=0,-\right)$. On examining the interaction vertices, by far the dominant annihilation processes are

$$
\phi \phi^{\dagger} \rightarrow N N, H_{u} H_{d}
$$

via the $t$ - and $u$-channel messenger exchange and $s$-channel singlet exchange. Following [21], we define a Lorentz invariant function

$$
w(s) \equiv \frac{1}{64 \pi} \int_{-1}^{1} \frac{1}{8} \sum_{Q, Q^{\prime}, a, b}\left|\mathcal{M}\left(\phi^{Q \dagger} \phi^{Q^{\prime}} \rightarrow a b\right)\right|^{2} d \cos \theta
$$




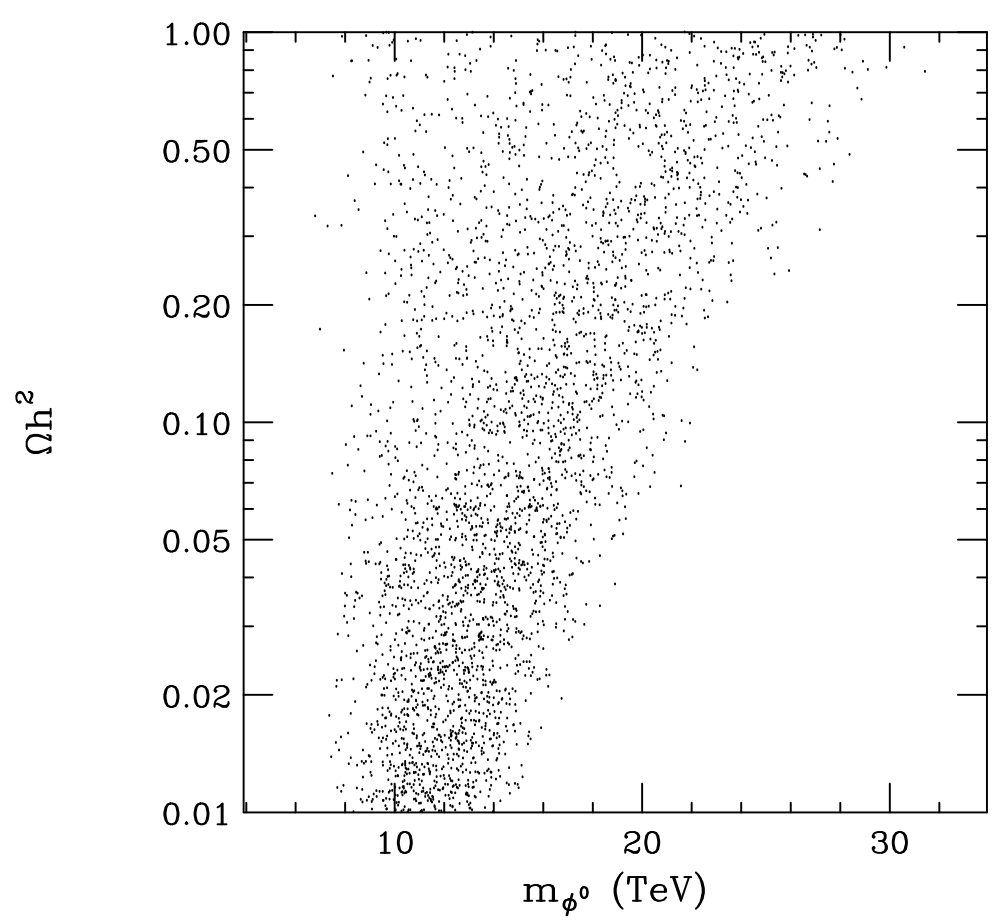

FIG. 5. Messenger sneutrino masses that do not over-close the universe.

where $\sqrt{s}$ is the center of mass energy. Specifically in our model,

$$
w(s)=\left(\frac{\left(\eta_{N} k\langle S\rangle\right)^{2} \vartheta}{16 \sqrt{\pi} s}\right)^{2}\left[1-\frac{1}{\sqrt{2}}+2\left(\frac{\lambda_{N}}{k}\right)^{2}-\frac{4}{3} \vartheta\left(1-\frac{m_{\phi}^{2}}{s}\right)+\vartheta^{2} \frac{s}{4 m_{\phi}^{2}}\right]
$$

where $\vartheta=2 \xi_{N}\left(2 \xi_{S}-\eta_{N}\right) /\left(\eta_{N} k\right)$. With $\kappa=T_{f} / m_{\phi^{0}}$ and

$$
A=w\left(4 m_{\phi^{0}}^{2}\right), \quad B=3\left[2 m_{\phi^{0}}^{2} \frac{\partial w(s)}{\partial s}-w(s)\right]_{s=4 m_{\phi^{0}}^{2}}
$$

the thermal average $\left\langle\bar{\sigma} v_{\text {rel }}\right\rangle$ can be expressed as

$$
\left\langle\bar{\sigma} v_{\mathrm{rel}}\right\rangle=\frac{1}{m_{\phi^{0}}^{2}}(A+B \kappa)
$$

It is customary to express the relic abundance in terms of the mass density in units of the critical density $\Omega_{\phi^{0}}=\rho_{\phi^{0}} / \rho_{c}$. It is found that

$$
\Omega_{\phi^{0}} h^{2}=\frac{8.5 \times 10^{-5}}{\sqrt{g_{*}}}\left(\frac{m_{\phi^{0}}}{\mathrm{TeV}}\right)^{2} \frac{\kappa^{-1}}{A+\frac{1}{2} B \kappa},
$$

where $g_{*} \simeq 232.5$ for the particle content of the NMSSM at the freeze-out temperature $T_{f}$. The value of $\kappa$ is related to $T_{f}$ and is obtained iteratively by

$$
\kappa^{-1} \equiv m_{\phi^{0}} / T_{f}=\ln \left[\frac{0.076}{\sqrt{g_{*}}} \frac{M_{P l}}{m_{\phi^{0}}}(A+B \kappa) \sqrt{\kappa}\right] .
$$


From the parameter space that provided a phenomenologically viable solution to the $\mu$ problem we select the subspace that does not over-close the Universe. Fig. 5 shows the relic density versus the cold dark matter mass $m_{\phi^{0}}$, where $m_{\phi^{0}} /\langle S\rangle$ was allowed to vary between 0.1 and 1 . If we assume that $\Omega_{\phi^{0}} h^{2} \sim 0.3$, then the mass of the lightest messenger sneutrino lies in the range

$$
6 \lesssim m_{\phi^{0}} \lesssim 25 \mathrm{TeV}
$$

The smaller values of $m_{\phi^{0}}$ correspond to $m_{\phi^{0}} /\langle S\rangle$ close to 0.1 and are undesirable from the fine-tuning argument. We conclude that massive scalar messenger cold dark matter is natural in this model.

\section{A RESOLUTION OF THE COSMOLOGICAL DOMAIN WALL PROBLEM}

It is well-known that the NMSSM predicts the formation of domain walls at the weak scale arising from the spontaneous breaking of the $\mathbf{Z}_{\mathbf{3}}$ symmetry of the superpotential. Although there are proposals to resolve this problem [22], the most straightforward solution still involves the introduction of higher dimensional non-renormalizable operators that explicitly break the discrete symmetry. It has been shown that this solution is unsatisfactory when SUSY-breaking is mediated by gravity [4]. We briefly indicate where the problem lies.

A wall network propagates once it has formed. The most important force acting on the domain walls that can dissolve them is pressure. Pressure arises from an explicit violation of the discrete symmetry. If the vacua are separated by an energy density $\epsilon$, the pressure is typically of order $\epsilon$. When this difference is greater than the surface tension $\sigma / R$, where $R$ is the curvature scale of the walls and $\sigma$ is the surface energy density, there is a minimum value of $\epsilon$ above which the dynamics will have been dominated by pressure before today. Thus, as the Universe expands, pressure catches up with surface tension, ultimately dominates it, the wall network is destroyed and the true vacuum is selected.

In general if the $\mathbf{Z}_{\mathbf{3}}$ symmetry is broken at an energy scale $v_{0}$, then $\sigma \sim v_{0}^{3}$, and if pressure is to be dominant at an epoch $T_{*}$ before the quark-hadron phase transition, the curvature scale will be roughly the scale factor $R \sim M_{P l} / T^{2}$, and the relation

$$
\epsilon \gtrsim \frac{v_{0}^{3} T_{*}^{2}}{M_{P l}}
$$

must hold. With $\phi$ a real scalar field having a VEV $v_{0}$, the leading higher dimensional $\mathbf{Z}_{\mathbf{3}}$-breaking operator can be written as $\lambda^{\prime} \phi^{5} / M_{P l}$. Assuming other couplings to be $\mathcal{O}(1)$, the above constraint can now be rephrased as a constraint on $\lambda^{\prime}$ :

$$
\lambda^{\prime} \gtrsim\left(\frac{T_{*}}{v_{0}}\right)^{2} .
$$

Note that the above relation holds only when all the fields in the $\mathbf{Z}_{\mathbf{3}}$-breaking operator acquire the same VEV as the scale at which the symmetry is broken. The relevance of this point will become clear below.

Nucleosynthesis dictates that the walls decay before the Universe cools to 0.1 to $1 \mathrm{MeV}$. If this were not the case, the abundances of the lighter elements would be significantly smaller than measured. With $T_{*} \sim 10 \mathrm{MeV}$ and $v_{0}=v \simeq 174 \mathrm{GeV}$, 


$$
\lambda^{\prime} \gtrsim 10^{-8}
$$

However, when the mediation of SUSY-breaking is gravitational, this leads to destabilization of the hierarchy [ [1,5]. For example, the operator $\lambda^{\prime}\left(H_{u} H_{d}\right) N^{2} / M_{P l}$ along with $\lambda_{N} H_{u} H_{d} N$ generates a tadpole that leads to a term in the superpotential of the form $\mu^{\prime} N$ with

$$
\mu^{\prime} \sim \frac{\lambda^{\prime} \lambda_{N}}{\left(16 \pi^{2}\right)^{2}} \Lambda_{\text {Gravity }}^{2}
$$

where $\Lambda_{\text {Gravity }} \sim \mathcal{O}\left(10^{10} \mathrm{GeV}\right)$ is the SUSY-breaking insertion. For $\lambda^{\prime} \gtrsim 10^{-8}$, this gives $\left(\mu^{\prime} x\right)^{1 / 3} \gtrsim 6 \mathrm{TeV}$ thus destabilizing the electroweak scale. In other words, to obtain $\mu^{\prime} x \sim \mathcal{O}\left(v^{3}\right)$, one needs $\lambda^{\prime} \lesssim 10^{-11}$, in obvious contradiction with cosmology.

In our model, the operator that can destabilize the hierarchy most severely is $\lambda^{\prime}\left(H_{u} H_{d}\right) S^{2} / M_{P l}$. The only field with a messenger scale VEV is $S$. The lower bound on $\lambda^{\prime}$ is now altered to

$$
\lambda^{\prime} \lambda_{S} \gtrsim\left(\frac{T_{*} v_{0}}{v^{2}}\right)^{2} \sim 10^{-3}
$$

for $v_{0}=y \sim 10^{5}$. With $\lambda_{S} \sim \mathcal{O}\left(10^{-3}\right)$, this leads $\lambda^{\prime}$ to the natural value $\lambda^{\prime} \sim \mathcal{O}(1)$. Such a large value of $\lambda^{\prime}$ looks a lot worse than in the gravity-mediated case in terms of the destabilization of the hierarchy. However, a crucial observation is that in GMSB models the SUSY-breaking scale is typically very low. In the model under consideration, SUSY-breaking is communicated via a direct interaction $\xi_{S} S \bar{\Phi} \Phi$ and consequently the fundamental scale of SUSY-breaking $F_{0}=F_{S} / \xi_{S} \sim \Lambda^{2}$ [8], where $\Lambda$ is 50-100 TeV. As earlier, the operators $\lambda^{\prime}\left(H_{u} H_{d}\right) S^{2} / M_{P l}$ and $\lambda_{S} H_{u} H_{d} S$ generate a term in the superpotential of the form $\mu^{\prime} S$. In this case,

$$
\mu^{\prime} \sim \frac{\lambda^{\prime} \lambda_{S}}{\left(16 \pi^{2}\right)^{2}} \Lambda^{2} \simeq\left(\frac{\lambda^{\prime} \lambda_{S}}{4 \times 10^{-3}}\right)\left(\frac{\Lambda}{100 \mathrm{TeV}}\right)^{2}(30 \mathrm{GeV})^{2}
$$

Therefore, the stability of the electroweak scale can be maintained, $\mu^{\prime} y \sim \mathcal{O}\left(v^{3}\right)$, while simultaneously satisfying the cosmological constraints. However, if the fundamental SUSYbreaking scale happens to be much higher $F_{0} \gg \Lambda^{2}$, the problem of destabilizing the electroweak scale will remain.

\section{DISCUSSION AND CONCLUSION}

By design in our model, both $\mu$ and $B_{\mu}$ parameters are generated spontaneously at the right order, thus solving the $\mu$-problem. The fine-tune for the cancellation between $\mu^{2}$ and $m_{H_{u}}^{2}$ to obtain the correct $M_{Z}$ is still at the percentage level, as in the MSSM. The primary reason that we are able to overcome the $\mu$-problem in GMSB [9] is the inclusion of messengermatter interactions in the superpotential. However, the smallness of the parameters $\eta_{S}$ and $\lambda_{S}$ should be further explored in terms of discrete symmetries arising from the SUSYbreaking sector. 
As for low-energy phenomenology, we see from Table 1 that the sparticle spectrum is typical of gauge-mediated models. It is worth mentioning that if we require $k$ to be perturbative only below the messenger scale $\Lambda$, we find $k \leq 1.36$. This results in the upper bound on the lightest Higgs boson mass to be about $170 \mathrm{GeV}$.

In summary, we studied in some detail the next-to-minimal supersymmetric standard model with the gauge mediation of supersymmetry breaking. We found that it is feasible to spontaneously generate the $\mu$ and $B_{\mu}$ terms which are consistent with electroweak symmetry breaking and the current sparticle mass limits. Messenger sneutrinos with mass in the range 6 to $25 \mathrm{TeV}$ can serve as cold dark matter. It is also possible to find a solution to the cosmological domain wall problem consistent with EWSB due to the low scale of SUSYbreaking

Acknowledgments: We thank H.-C. Cheng and M. Drees for helpful comments on the manuscript. This work was supported in part by a DOE grant No. DE-FG02-95ER40896 and in part by the Wisconsin Alumni Research Foundation.

\section{APPENDIX A:}

The 1-loop renormalization group equations of the couplings and soft-SUSY breaking terms relevant for our analysis are listed. We only include contributions from the third generation to the superpotential and soft-terms.

$$
\begin{array}{rl}
W=\xi_{S} & S \Phi \bar{\Phi}+\xi_{N} N \Phi \bar{\Phi}-\frac{\eta_{N}}{2} N^{2} S-\frac{\eta_{S}}{2} S^{2} N+\lambda_{N} N H_{u} H_{d} \\
& +\lambda_{S} S H_{u} H_{d}-\frac{k}{3} N^{3}+h_{t} H_{u} Q \bar{U}+h_{b} H_{d} Q \bar{D}+h_{\tau} H_{d} L \bar{E} \\
V_{\text {soft }}=m_{H_{d}}^{2}\left|H_{d}\right|^{2}+m_{H_{u}}^{2}\left|H_{u}\right|^{2}+m_{N}^{2}|N|^{2}+m_{\tilde{t}_{L}}^{2}\left|\tilde{t}_{L}\right|^{2}+m_{\tilde{t}_{R}}^{2}\left|\tilde{t}_{R}\right|^{2}+m_{\tilde{b}_{R}}^{2}\left|\tilde{b}_{R}\right|^{2}+m_{\tilde{\tau}_{L}}^{2}\left|\tilde{\tau}_{L}\right|^{2} \\
\quad+m_{\tilde{\tau}_{R}}^{2}\left|\tilde{\tau}_{R}\right|^{2}+\frac{1}{2} M_{1} \tilde{B} \tilde{B}+\frac{1}{2} M_{2} \tilde{W} \tilde{W}+\frac{1}{2} M_{3} \tilde{g} \tilde{g}+\left(A_{t} h_{t} \tilde{t}_{L} \tilde{t}_{R} H_{u}+A_{b} h_{b} \tilde{b}_{L} \tilde{b}_{R} H_{d}\right. \\
\left.\quad+A_{\tau} h_{\tau} \tilde{\tau}_{L} \tilde{\tau}_{R} H_{d}-\lambda_{N} A_{\lambda_{N}} H_{d} H_{u} N-\frac{k}{3} A_{k} N^{3}+h . c .\right) .
\end{array}
$$

For the gauge and Yukawa couplings:

$$
\begin{aligned}
16 \pi^{2} \frac{d}{d t} g^{\prime} & =11 g^{\prime 3}, \quad 16 \pi^{2} \frac{d}{d t} g_{2}=g_{2}^{3}, \quad 16 \pi^{2} \frac{d}{d t} g_{3}=-3 g_{3}^{3} \\
16 \pi^{2} \frac{d}{d t} h_{t} & =h_{t}\left(6 h_{t}^{2}+h_{b}^{2}+\lambda_{N}^{2}+\lambda_{S}^{2}-\frac{13}{9} g^{\prime 2}-3 g_{2}^{2}-\frac{16}{3} g_{3}^{2}\right) \\
16 \pi^{2} \frac{d}{d t} h_{b} & =h_{b}\left(6 h_{b}^{2}+h_{t}^{2}+h_{\tau}^{2}+\lambda_{N}^{2}+\lambda_{S}^{2}-\frac{7}{9} g^{\prime 2}-3 g_{2}^{2}-\frac{16}{3} g_{3}^{2}\right), \\
16 \pi^{2} \frac{d}{d t} h_{\tau} & =h_{\tau}\left(4 h_{\tau}^{2}+3 h_{b}^{2}+\lambda_{S}^{2}-3 g^{\prime 2}-3 g_{2}^{2}\right) .
\end{aligned}
$$


For the couplings particular to the NMSSM and to our model:

$$
\begin{aligned}
16 \pi^{2} \frac{d}{d t} k & =6 k\left(k^{2}+\lambda_{N}^{2}\right)-\eta_{N}^{2}\left(2 k+\eta_{S}\right) \\
16 \pi^{2} \frac{d}{d t} \lambda_{N} & =\lambda_{N}\left(2 \lambda_{S}^{2}+\eta_{S}^{2}+\eta_{N}^{2}+5 \xi_{N}^{2}+4 \lambda_{N}^{2}+2 k^{2}+3 h_{t}^{2}+3 h_{b}^{2}+h_{\tau}^{2}-g^{2}-3 g_{2}^{2}\right) \\
16 \pi^{2} \frac{d}{d t} \lambda_{S} & =\lambda_{S}\left(2 \lambda_{N}^{2}+4 \lambda_{S}^{2}+\eta_{S}^{2}+\eta_{N}^{2}+5 \xi_{S}^{2}+3 h_{t}^{2}+3 h_{b}^{2}+h_{\tau}^{2}-g^{2}-3 g_{2}^{2}\right) \\
16 \pi^{2} \frac{d}{d t} \xi_{N} & =\xi_{N}\left(9 \xi_{N}^{2}+4 \xi_{S}^{2}+\eta_{S}^{2}+\eta_{N}^{2}+2 \lambda_{N}^{2}+2 k^{2}+\frac{13}{9} g^{2}-3 g_{2}^{2}-\frac{16}{3} g_{3}^{2}\right) \\
16 \pi^{2} \frac{d}{d t} \xi_{S} & =\xi_{S}\left(9 \xi_{S}^{2}+4 \xi_{N}^{2}+\eta_{S}^{2}+\eta_{N}^{2}+2 \lambda_{S}^{2}+\frac{13}{9} g^{\prime 2}-3 g_{2}^{2}-\frac{16}{3} g_{3}^{2}\right) \\
16 \pi^{2} \frac{d}{d t} \eta_{N} & =\eta_{N}\left(5 \xi_{S}^{2}+10 \xi_{N}^{2}+3 \eta_{S}^{2}+3 \eta_{N}^{2}+2 \lambda_{S}^{2}+4 \lambda_{N}^{2}+4 k^{2}\right) \\
16 \pi^{2} \frac{d}{d t} \eta_{S} & =\eta_{S}\left(5 \xi_{N}^{2}+10 \xi_{S}^{2}+3 \eta_{S}^{2}+3 \eta_{N}^{2}+4 \lambda_{S}^{2}+2 \lambda_{N}^{2}+2 k^{2}\right)
\end{aligned}
$$

For the trilinear couplings of the third generation:

$$
\begin{aligned}
16 \pi^{2} \frac{d}{d t} A_{t} & =12 h_{t}^{2} A_{t}+2 h_{b}^{2} A_{b}+2 \lambda_{N}^{2} A_{\lambda}-4\left(\frac{13}{18} g^{\prime 2} M_{1}+\frac{3}{2} g_{2}^{2} M_{2}+\frac{8}{3} g_{3}^{2} M_{3}\right) \\
16 \pi^{2} \frac{d}{d t} A_{b} & =12 h_{b}^{2} A_{b}+2 h_{t}^{2} A_{t}+2 h_{\tau}^{2} A_{\tau}+2 \lambda_{N}^{2} A_{\lambda}-4\left(\frac{7}{18} g^{\prime 2} M_{1}+\frac{3}{2} g_{2}^{2} M_{2}+\frac{8}{3} g_{3}^{2} M_{3}\right) \\
16 \pi^{2} \frac{d}{d t} A_{\tau} & =8 h_{\tau}^{2} A_{\tau}+6 h_{b}^{2} A_{b}+2 \lambda_{N}^{2} A_{\lambda}-6\left(g^{\prime 2} M_{1}+g_{2}^{2} M_{2}\right) \\
16 \pi^{2} \frac{d}{d t} A_{\lambda} & =8 \lambda_{N}^{2} A_{\lambda}+2 \lambda_{S}^{2} A_{\lambda}-4 k^{2} A_{k}+6 h_{t}^{2} A_{t}+6 h_{b}^{2} A_{b}+2 h_{\tau}^{2}-2\left(g^{\prime 2} M_{1}+3 g_{2}^{2} M_{2}\right) \\
16 \pi^{2} \frac{d}{d t} A_{k} & =12\left(k^{2} A_{k}-\lambda_{N}^{2} A_{\lambda}\right)+\eta_{N}^{2} A_{k} .
\end{aligned}
$$

For the soft masses:

$$
\begin{gathered}
16 \pi^{2} \frac{d}{d t} M_{1}=22 g^{\prime 2} M_{1}, \quad 16 \pi^{2} \frac{d}{d t} M_{2}=2 g_{2}^{2} M_{2}, \quad 16 \pi^{2} \frac{d}{d t} M_{3}=-6 g_{3}^{2} M_{3}, \\
16 \pi^{2} \frac{d}{d t} m_{\tilde{t}_{L}}^{2}=2 h_{t}^{2}\left(m_{\tilde{t}_{L}}^{2}+m_{H_{u}}^{2}+m_{\tilde{t}_{R}}^{2}+A_{t}^{2}\right)+2 h_{b}^{2}\left(m_{\tilde{t}_{L}}^{2}+m_{H_{d}}^{2}+m_{\tilde{b}_{R}}^{2}+A_{b}^{2}\right) \\
\quad+\frac{1}{3} g^{\prime 2} \zeta-8\left(\frac{1}{36} g^{\prime 2} M_{1}^{2}+\frac{3}{4} g_{2}^{2} M_{2}^{2}+\frac{4}{3} g_{3}^{2} M_{3}^{2}\right), \\
16 \pi^{2} \frac{d}{d t} m_{\tilde{t}_{R}}^{2}=4 h_{t}^{2}\left(m_{\tilde{t}_{L}}^{2}+m_{H_{u}}^{2}+m_{\tilde{t}_{R}}^{2}+A_{t}^{2}\right)-8\left(\frac{4}{9} g^{\prime 2} M_{1}^{2}+\frac{4}{3} g_{3}^{2} M_{3}^{2}\right)-\frac{4}{3} g^{\prime 2} \zeta, \\
16 \pi^{2} \frac{d}{d t} m_{\tilde{b}_{R}}^{2}=4 h_{b}^{2}\left(m_{\tilde{Q}_{3}}^{2}+m_{H_{d}}^{2}+m_{\tilde{b}}^{2}+A_{b}^{2}\right)-8\left(\frac{1}{9} g^{\prime 2} M_{1}^{2}+\frac{4}{3} g_{3}^{2} M_{3}^{2}\right)+\frac{2}{3} g^{\prime 2} \zeta, \\
16 \pi^{2} \frac{d}{d t} m_{\tilde{\tau}_{L}}^{2}=2 h_{\tau}^{2}\left(m_{\tilde{\tau}_{L}}^{2}+m_{H_{d}}^{2}+m_{\tilde{\tau}_{R}}^{2}+A_{\tau}^{2}\right)-8\left(\frac{1}{4} g^{\prime 2} M_{1}^{2}+\frac{3}{4} g_{2}^{2} M_{2}^{2}\right)-g^{\prime 2} \zeta, \\
16 \pi^{2} \frac{d}{d t} m_{\tilde{\tau}_{R}}^{2}=4 h_{\tau}^{2}\left(m_{\tilde{\tau}_{L}}^{2}+m_{H_{d}}^{2}+m_{\tilde{\tau}_{R}}^{2}+A_{\tau}^{2}\right)-8 g^{\prime 2} M_{1}^{2}+2 g^{\prime 2} \zeta, \\
16 \pi^{2} \frac{d}{d t} m_{H_{d}}^{2}=6 h_{b}^{2}\left(m_{H_{d}}^{2}+m_{\tilde{t}_{L}}^{2}+m_{\tilde{b}_{R}}^{2}+A_{b}^{2}\right)+2 \lambda_{S}^{2}\left(m_{H_{d}}^{2}+m_{H_{u}}^{2}\right)
\end{gathered}
$$




$$
\begin{gathered}
+2 h_{\tau}^{2}\left(m_{H_{d}}^{2}+m_{\tilde{\tau}_{L}}^{2}+m_{\tilde{\tau}_{R}}^{2}+A_{\tau}^{2}\right)+2 \lambda_{N}^{2}\left(m_{H_{d}}^{2}+m_{H_{u}}^{2}+m_{N}^{2}+A_{\lambda}^{2}\right) \\
-8\left(\frac{1}{4} g^{\prime 2} M_{1}^{2}+\frac{3}{4} g_{2}^{2} M_{2}^{2}\right)-g^{\prime 2} \zeta \\
16 \pi^{2} \frac{d}{d t} m_{H_{u}}^{2}=6 h_{t}^{2}\left(m_{H_{u}}^{2}+m_{\tilde{t}_{L}}^{2}+m_{\tilde{t}_{R}}^{2}+A_{t}^{2}\right)+2 \lambda_{S}^{2}\left(m_{H_{d}}^{2}+m_{H_{u}}^{2}\right) \\
-8\left(\frac{1}{4} g^{\prime 2} M_{1}^{2}+\frac{3}{4} g_{2}^{2} M_{2}^{2}\right)+2 \lambda_{N}^{2}\left(m_{H_{d}}^{2}+m_{H_{u}}^{2}+m_{N}^{2}+A_{\lambda}^{2}\right)+g^{\prime 2} \zeta, \\
16 \pi^{2} \frac{d}{d t} m_{N}^{2}=4 \lambda_{N}^{2}\left(m_{N}^{2}+m_{H_{u}}^{2}+m_{H_{d}}^{2}+A_{\lambda}^{2}\right)+4 k^{2}\left(3 m_{N}^{2}+A_{k}^{2}\right),
\end{gathered}
$$

where $\zeta=\sum_{i} Y_{i} m_{i}^{2}$ is the hypercharge-weighted sum of the squares of the soft masses. As a consequence of the boundary conditions at the messenger scale, $\zeta=0$ at all energies.

\section{APPENDIX B:}

The squared mass matrices for the neutral and charged Higgses are presented. The analytic expressions for their diagonalization are displayed.

$$
M_{C P \text {-even }}^{2}=\frac{1}{2} \frac{\partial^{2} V_{\text {neutral }}^{1-\text { loop }}}{\partial v_{i} \partial v_{j}}=\frac{1}{2}\left(\begin{array}{lll}
a & d & e \\
d & b & f \\
e & f & c
\end{array}\right),
$$

where $v_{1}=v_{d}, v_{2}=v_{u}, v_{3}=x$ and

$$
\begin{array}{rl}
a= & \frac{1}{v_{d}}\left(\bar{g}^{2} v_{d}^{3}+v_{u}\left(\left(2 k x^{2}+2 x A_{\lambda_{N}}+2 x y \eta_{N}+y^{2} \eta_{S}\right) \lambda_{N}+\left(-2 F_{S}+x^{2} \eta_{N}+2 x y \eta_{S}\right) \lambda_{S}\right)\right) \\
& -\left(\partial_{v_{d}} \Delta V\right) / v_{d}+\partial_{v_{d}, v_{d}}^{2} \Delta V \\
b= & \frac{1}{v_{u}}\left(\bar{g}^{2} v_{u}^{3}+v_{d}\left(\left(2 k x^{2}+2 x A_{\lambda_{N}}+2 x y \eta_{N}+y^{2} \eta_{S}\right) \lambda_{N}+\left(-2 F_{S}+x^{2} \eta_{N}+2 x y \eta_{S}\right) \lambda_{S}\right)\right) \\
& -\left(\partial_{v_{u}} \Delta V\right) / v_{u}+\partial_{v_{u}, v_{u}}^{2} \Delta V \\
c= & \frac{1}{8 \pi^{2} x y}\left(-16 k \pi^{2} x^{2} y A_{k}-F_{S}^{2} \xi_{N} \xi_{S}+16 \pi^{2} y^{2} F_{S} \eta_{S}+8 \pi^{2} y\left(y \eta _ { N } \left(6 k x^{2}+\left(3 x^{2}-y^{2}\right) \eta_{S}\right.\right.\right. \\
& \left.\left.\left.+2 v_{d} v_{u} \lambda_{N}\right)+2 x^{3} \eta_{N}^{2}+2\left(4 k^{2} x^{3}+A_{\lambda_{N}} v_{d} v_{u} \lambda_{N}+y v_{d} v_{u} \eta_{S} \lambda_{S}-y v_{d}^{2} \lambda_{N} \lambda_{S}-y v_{u}^{2} \lambda_{N} \lambda_{S}\right)\right)\right) \\
& -\left(\partial_{x} \Delta V\right) / x+\partial_{x, x}^{2} \Delta V \\
d= & -\left(2 k x^{2}+2 x A_{\lambda_{N}}+2 x y \eta_{N}+y^{2} \eta_{S}\right) \lambda_{N}+\lambda_{S}\left(2 F_{S}-x^{2} \eta_{N}-2 x y \eta_{S}+4 v_{d} v_{u} \lambda_{S}\right) \\
& -\bar{g}^{2} v_{d} v_{u}+4 v_{d} v_{u} \lambda_{N}^{2}+\partial_{v_{d}, v_{u}}^{2} \Delta V \\
e & 4 v_{d} \lambda_{N}\left(x \lambda_{N}+y \lambda_{S}\right)-2 v_{u}\left(\left(2 k x+A_{\lambda_{N}}+y \eta_{N}\right) \lambda_{N}+\left(x \eta_{N}+y \eta_{S}\right) \lambda_{S}\right)+\partial_{v_{d}, x}^{2} \Delta V \\
f= & 4 v_{u} \lambda_{N}\left(x \lambda_{N}+y \lambda_{S}\right)-2 v_{d}\left(\left(2 k x+A_{\lambda_{N}}+y \eta_{N}\right) \lambda_{N}+\left(x \eta_{N}+y \eta_{S}\right) \lambda_{S}\right)+\partial_{v_{u}, x}^{2} \Delta V .
\end{array}
$$

The eigenvalue equation from this matrix is

$$
\left(m^{2}\right)^{3}+a_{2}\left(m^{2}\right)^{2}+a_{1} m^{2}+a_{0}=0
$$

where 


$$
\begin{aligned}
& a_{0}=-a b c-2 d e f+a f^{2}+b e^{2}+c d^{2} \\
& a_{1}=a b+a c+b c-\left(d^{2}+e^{2}+f^{2}\right) \\
& a_{2}=-(a+b+c) .
\end{aligned}
$$

With

$$
r=\frac{1}{6}\left(a_{1} a_{2}-3 a_{0}\right)-\frac{1}{27} a_{2}^{3}, \quad q=-\frac{1}{3} a_{1}+\frac{1}{9} a_{2}^{2}, \quad \theta=\frac{1}{3} \arccos \frac{r}{q^{3 / 2}},
$$

the eigenvalues are

$$
\begin{aligned}
& m_{S_{1}}^{2}=-\sqrt{q}(\cos \theta+\sqrt{3} \sin \theta)-\frac{a_{2}}{3} \\
& m_{S_{2}}^{2}=\sqrt{q}(-\cos \theta+\sqrt{3} \sin \theta)-\frac{a_{2}}{3} \\
& m_{S_{3}}^{2}=2 \sqrt{q} \cos \theta-\frac{a_{2}}{3} .
\end{aligned}
$$

Now for the CP-odd Higgs bosons. The mass squared matrix is

$$
M_{C P-o d d}^{2}=\left(\begin{array}{ll}
r & j \\
j & s
\end{array}\right)
$$

where

$$
\begin{aligned}
& r=\frac{v^{2}}{2 v_{d} v_{u}}\left(\left(2 k x^{2}+2 x A_{\lambda_{N}}+2 x y \eta_{N}+y^{2} \eta_{S}\right) \lambda_{N}+\left(-2 F_{S}+x\left(x \eta_{N}+2 y \eta_{S}\right)\right) \lambda_{S}\right) \\
& s=\frac{1}{16 \pi^{2} x y}\left[48 k \pi^{2} x^{2} y A_{k}-F_{S}^{2} \xi_{N} \xi_{S}+16 \pi^{2} y F_{S}\left(2 x \eta_{N}+y \eta_{S}\right)-8 \pi^{2} y\left(\eta _ { N } \left(2 k x^{2} y+y\left(x^{2}+y^{2}\right) \eta_{S}\right.\right.\right. \\
& \left.\left.\left.-2 v_{d} v_{u}\left(y \lambda_{N}+2 x \lambda_{S}\right)\right)+2\left(y \eta_{S}\left(2 k x y-v_{d} v_{u} \lambda_{S}\right)+\lambda_{N}\left(\left(-4 k x-A_{\lambda_{N}}\right) v_{d} v_{u}+y v^{2} \lambda_{S}\right)\right)\right)\right] \\
& j=-\left(v\left(\left(2 k x-A_{\lambda_{N}}+y \eta_{N}\right) \lambda_{N}+\left(x \eta_{N}+y \eta_{S}\right) \lambda_{S}\right)\right) .
\end{aligned}
$$

The transformation matrix that takes us from the $\left(A^{0} \equiv \sin \beta \operatorname{Im} H_{d}+\cos \beta \operatorname{Im} H_{u}\right)-\operatorname{Im} N$ basis to the diagonal basis is

$$
\left(\begin{array}{cc}
\cos \gamma & \sin \gamma \\
-\sin \gamma & \cos \gamma
\end{array}\right)
$$

where

$$
\sin 2 \gamma=\frac{-2 j}{\sqrt{(s-r)^{2}+4 j^{2}}}, \quad \cos 2 \gamma=\frac{s-r}{\sqrt{(s-r)^{2}+4 j^{2}}}
$$

and the eigenvalues are

$$
m_{P_{1}, P_{2}}^{2}=\frac{1}{2}\left(s+r \mp \sqrt{(s-r)^{2}+4 j^{2}}\right) .
$$

Finally, the squared mass of the charged Higgs is

$$
m_{H^{ \pm}}^{2}=M_{W}^{2}-\left(\lambda_{N}^{2}+\lambda_{S}^{2}\right) v^{2}+r
$$

where $r$ is the first entry of the CP-odd Higgs matrix. 


\section{REFERENCES}

[1] See, e.g., H.P. Nilles, Phys. Rept. 110, 1 (1984); H.E. Haber and G.L. Kane, Phys. Rept. 117, 75 (1985).

[2] H.P. Nilles, M. Srednicki and D. Wyler, Phys. Lett. B120, 346 (1983); J.M. Frere, D.R.T. Jones and S. Raby, Nucl. Phys. B222, 11 (1983); J.P. Derendinger and C. Savoy, Nucl. Phys. B237, 307 (1984); L.E. Ibanez and J. Mas, Nucl. Phys. B286, 107 (1987); J. Ellis, J. Gunion, H. Haber, L. Roskowski and F. Zwirner, Phys. Rev. D39, 844 (1989); M. Drees, Int. J. Mod. Phys. A4, 3635 (1989); U. Ellwanger, M. Rausch de Traubenberg and C.A. Savoy, Nucl. Phys. B492, 21 (1997).

[3] J.E. Kim and H.P. Nilles, Phys. Lett. B138, 150 (1984); G.F. Giudice and A. Masiero, Phys. Lett. B206, 480 (1988); E.J. Chun, J.E. Kim and Nilles, Nucl. Phys. B370, 105 (1992); J. Casas and C. Munoz, Phys. Lett. B306, 188 (1993).

[4] S. Abel, S. Sarkar and P. White, Nucl. Phys. B454, 663 (1995).

[5] U. Ellwanger, Phys. Lett. B133, 187 (1983); J. Bagger and E. Poppitz, Phys. Rev. Lett. 71, 2380 (1993); V. Jain, Phys. Lett. B351, 481 (1995); J. Bagger, E. Poppitz and L. Randall, Nucl. Phys. B455, 59 (1995).

[6] M. Dine, A. E. Nelson and Y. Shirman, Phys. Rev. D51, 1362 (1995).

[7] M. Dine and A. Nelson, Phys. Rev. D48, 1277 (1993); M. Dine, A. Nelson, Y. Nir and Y. Shirman, Phys. Rev. D53, 2658 (1996);

[8] G. Giudice and R. Rattazzi, hep-ph/9801271; S.L. Dubovsky, D.S. Gorbunov and S.V. Troitsky, hep-ph/9905466; and references therein; G. Giudice and R. Rattazzi, Nucl. Phys. B511, 25 (1998).

[9] G. Dvali, G. Giudice and A. Pomarol, Nucl. Phys. B478, 31 (1996); T. Yanagida, Phys. Lett. B400, 109 (1997); P. Langacker, N. Polonsky and J. Wang, hep-ph/9905252.

[10] A. de Gouvêa, A. Friedland and H. Murayama, Phys. Rev. D57, 5676 (1998).

[11] S. Martin and M. Vaughn, Phys. Rev. D50, 2282 (1994); Y. Yamada, Phys. Rev. D50, 3537 (1994).

[12] S. Lammel, Fermilab-CONF-98-055-E, hep-ex/9802010.

[13] ALEPH Collaboration, ALEPH 99-006; DELPHI Collaboration, DELPHI 99-142.

[14] R. Barbieri and G. F. Giudice, Nucl. Phys. B306, 63 (1988).

[15] K. Agashe and M. Graesser, Nucl. Phys. B507, 3 (1997).

[16] K. Babu, C. Kolda and F. Wilczek, Phys. Rev. Lett. 77, 3070 (1997); S. Dimopoulos, S. Thomas and J. Wells, Nucl. Phys. B488, 39 (1997); J. Bagger, K. Matchev, D. Pierce and R.-J. Zhang, Phys. Rev. D55, 3188 (1997).

[17] H.E. Haber and Y. Nir, Nucl. Phys. B335, 363 (1990).

[18] S. Dimopoulos, G. F. Giudice and A. Pomarol, Phys. Lett. B389, 37 (1996).

[19] T. Han and R. Hempfling, Phys. Lett. B415, 161 (1997).

[20] T. Falk, K. A. Olive and M. Srednicki, Phys. Lett. B339, 248 (1994).

[21] M. Srednicki, R. Watkins and K. A. Olive, Nucl. Phys. B310, 693 (1988).

[22] J. McDonald, Nucl. Phys. B530, 325 (1998); C. Panagiotakopoulos and K. Tamvakis, Phys. Lett. B446, 224 (1999). 